\title{
Uma proposta de padrão de metadados para objetos de aprendizagem de museus de ciências e tecnologia*
}

\author{
Ana Carolina Bertoletti-De-Marchi ${ }^{* *}$ \\ Antônio Carlos da Rocha Costa ${ }^{* * * *}$
}

\begin{abstract}
Resumo: Este artigo tem como objetivo apresentar uma proposta de padrão de metadados para objetos de aprendizagem de museus de ciências e tecnologia. Os museus estão entre os espaços educacionais que contribuem para o crescimento de novas fontes eletrônicas de informações voltadas para a aprendizagem. De modo a disponibilizar tais informações ao público internauta e possibilitar também a troca entre instituições, estão sendo implantados em museus ao redor do mundo padrões de metadados. A maioria dos padrões já desenvolvidos não tem como principal preocupação a criação de metadados para a aprendizagem. Como o objetivo deste trabalho é promover a aprendizagem através das informações disponíveis nos museus, este artigo irá apresentar uma proposta de um padrão de metadados para descrever os objetos de aprendizagem de museus. O padrão denomina-se paDrão de metAdados para objeTos de Aprendizagem de Museus de Ciências e Tecnologia - DATA/MCT e está sendo atualmente validado no Museu Zoobotânico Agusto Ruschi.
\end{abstract}

Palavras-chaves: objetos de aprendizagem, metadados e padrões de metadados para museus.

A proposal of metadata standard for learning objects of museums of sciences and technology

\begin{abstract}
This paper intends to present a proposal of metadata standard for learning objects of museums of sciences and technology. The museums are among the educational spaces that contribute to the growth of new electronic sources of learning information. In way to offer such information to the public and make available to others institutions, they are being implanted in museums around the world metadata standard. Most of the standard already developed he/she doesn't have as main concern to create metadata for the learning. As the objective of this work is to promote the learning through the available information in the museums, this paper will present a proposal of a metadata standard to describe the learning objects of museums. The standard is called metadata standard for learning object of museums of sciences and technology and it is being validated now at the Muzar.
\end{abstract}

Keywords: learning object, metadata and metadata standard for museum.

\section{Introdução}

A grande procura de informações no WWW por estudantes, escolas, professores e pesquisadores tem mudado significativamente os recursos de informações digitais disponíveis na rede. Essa mudança se deve principalmente as novas fontes de referências de materiais que estão sendo disponibilizadas a fim de acrescentar ainda mais a teia mundial, como é o caso de, por exemplo, bibliotecas, coleções visuais e coleções científicas.

Entre os espaços educacionais que estão contribuindo para o crescimento de novas fontes eletrônicas estão os museus, que pela sua estruturação específica e pela riqueza de seu acervo, constituem-se em um espaço educativo não-formal que complementa a aprendizagem, tanto de alunos como do público em geral.

Segundo Trant et al. (2002), essa nova diversidade de fontes eletrônicas oferece muitas vantagens aos museus, como: a freqüente atualização, a possibilidade de suportar

\footnotetext{
* Artigo elaborado a partir da tese de doutorado em informática na educação (PGIE) com o apoio do CNPq através do edital MCT/SECIS/CNPq 7/2003.

** Professora, doutoranda em Informática na Educação (PGIE/UFRGS), professora e pesquisadora da UPF, carolina@upf.br. http://vitoria.upf.br/ carolina

**** Professor, Doutor em Ciência da Computação, professor e pesquidor da UCPel, rocha@ atlas.ucpel.tche.br.
} 
múltiplos usuários, o acesso sem considerar as horas em que está aberto, a oportunidade de aperfeiçoamento por parte dos leitores e o acesso a informações por pesquisadores.

Os museus criam e mantém uma variedade muito rica de informações descritivas para suportar uma grande gama de atividades que abrangem exibições, educação, programas públicos, pesquisa e conservação. Essas informações geralmente são armazenadas usando um software próprio de cada departamento da instituição e, muitas delas, não são acessíveis ao público. Com o passar do tempo essas informações tornamse redundantes nos gerenciadores de banco de dados.

Neste sentido, de modo a disponibilizar tais informações ao público internauta e possibilitar, também, a troca entre instituições, visando uma interoperabilidade de dados, estão sendo estudados e implantados padrões de metadados para gerenciamento de informações. Com o avanço tecnológico e os padrões de informações, os museus estão aptos a desenvolverem um novo modelo para indexar informações. A possibilidade de migrar informações de um sistema para outro em um formato independente, permite finalmente que os museus se aproximem do poderoso gerenciamento de informações, fazendo também com que o público tenha acesso facilitado as mesmas.

Atualmente, existem muitos padrões utilizados para gerenciar informações de coleções científicas e didáticas de museus. Esses padrões, que variam de padrões técnicos precisos a diretrizes gerais, permitem que os dados dos museus sejam eficiente e consistentemente indexados, armazenados, recuperados e compartilhados por diversos sistemas. Segundo CHIN (2003), os museus estão utilizando esses padrões para ajudálos a definir que tipos de informações registrar em seus bancos de dados (ou fichário) e estruturar essas informações (os relacionamentos entre diferentes tipos de informações).

Com a implementação desses padrões em museus, é possível a troca de dados entre outros sistemas e o compartilhamento com outras instituições, já que tais padrões se preocupam em armazenar as informações semânticas dos objetos.

Os pesquisadores de museus desejam usar suas coleções de dados para muitas aplicações, entre elas: catálogos de exibições, acesso público na Web e pesquisas. Com um padrão de metadados definido, essas coleções podem, além de compartilhar dados com outros museus, constituírem uma grande biblioteca virtual de informações para fins educacionais. Essa biblioteca poderia ser utilizada por um público diversificado como fonte de pesquisa e estudo.

Esse nível de interoperabilidade entre sistemas de museus requer que padrões de metadados sejam definidos e implementados. Os padrões permitem a troca de dados entre catálogos, pesquisas, entre organizações e entre sistema de computação, além de possibilitarem a busca semântica de informações.

Como a preocupação deste trabalho é promover a aprendizagem através das informações disponíveis nos museus, este artigo irá apresentar uma proposta de um padrão de metadados para descrever os objetos de aprendizagem dos museus. $\mathrm{O}$ padrão denomina-se paDrão de metAdados para objeTos de Aprendizagem de Museus de Ciências e Tecnologia - DATA/MCT e atualmente está sendo validado pelo Museu Zoobotânio Augusto Ruschi - UPF, onde o ambiente será implantado com apoio do CNPq.

\section{Metadados}

Metadados, ou dados sobre dados, funcionam de forma semelhante a um catálogo de biblioteca. Eles fornecem informações sobre um determinado recurso, promovendo a interoperabilidade, identificação, compartilhamento, integração, 
utilização, reutilização, gerenciamento e recuperação dos mesmos de maneira mais eficiente. São dados descritivos que podem informar sobre o título, autor, data, publicação, palavras-chaves, descrição, localização do recurso, entre outros. Eles podem ser comparados a um sistema de rotulagem que descreve o recurso, seus objetivos e características, mostrando como, quando e por quem o recurso foi armazenado, e como está formatado. Metadados são essenciais para entender o recurso armazenado, eles descrevem informações semânticas sobre o recurso.

Padrões de metadados para gerenciar e documentar coleções de museus estão sendo amplamente utilizados a fim de suportar suas necessidades, mantendo a consistência da base de dados com estratégias eficientes de pesquisa e, principalmente, promovendo a interoperabilidade entre sistemas.

O uso de padrões na documentação de coleções faz como que a troca de dados entre os museus que utilizam o mesmo padrão torne-se mais fácil, permitindo o compartilhamento de dados. Muitos padrões ao redor do mundo estão sendo desenvolvidos para esta finalidade. De acordo com o projeto CHIN (2003), estes padrões geralmente apresentam os seguintes objetivos:

- auxiliar na recuperação automática de informação;

- promover a consistência entre bancos de dados;

- assegurar que informações importantes sejam registradas;

- melhorar a segurança das coleções;

- ajudar os museus a respondem pelas suas coleções;

- promover o uso e o compartilhamento das coleções e conhecimentos;

- facilitar a troca de informação entre bancos de dados; e

- facilitar a migração de dados para sistemas novos.

$\mathrm{Na}$ área educacional os metadados são utilizados para descrever objetos de aprendizagem, ou seja recursos instrucionais que podem ser reutilizados por diversos ambientes, permitindo a apresentação e a rápida recuperação de acordo com as necessidades do contexto educacional que se está trabalhando. A proposta destes padrões é, além de facilitar o compartilhamento e a troca de objetos, possibilitar o desenvolvimento de catálogos e invenções enquanto consideram a diversidade de culturas e línguas em que os objetos de aprendizagem e seus metadados serão explorados (LTSC, 2002).

Neste trabalho, foram estudados vários padrões de metadados para museus e objetos de aprendizagem. Ambos os padrões de metadados, alcançam seus objetivos pois (CHIN, 2003):

- esboçam as unidades de informação que precisam ser registradas para documentar uma coleção corretamente (estruturam dados ou padrões de metadados);

- promovem um formato e convenções para armazenar as informações (conteúdo de dados ou padrões);

- $\quad$ sugerem fontes de terminologia (avaliam dados ou padrões de vocabulário).

Metadados para museus estão sendo largamente difundidos em países como Estados Unidos, Canadá e Reino Unido e, em especial, para registrar informações sobre os artefatos em exposição, sem explorar mais profundamente sua finalidade educacional. 
Para o desenvolvimento desta proposta foram pesquisados padrões que podem ser divididos em metadados para museus e educacionais. Os padrões de metadados para museus preocupam-se em documentar os objetos visando a interoperabilidade e a documentação em si e serão apresentados nas próximas seções. Por sua vez, os padrões educacionais estudados e utilizados na definição da proposta deste trabalho foram o LOM da IEEE e o Dublic Core, que pela sua grande utilização não serão apresentados aqui.

\subsection{Metadados para Museus}

\subsubsection{SPECTRUM (http://www.mda.org.uk/spectrum.htm)}

O SPECTRUM é um padrão de documentação dos museus do UK que representa um entendimento comum para uma boa documentação para museus, estabelecendo uma cooperação com a comunidade de museus.

Ele contém procedimentos para documentar objetos e os processos que eles sofrem, como também identificar e descrever informações que necessitam ser registradas para suportar os procedimentos.

O SPECTRUM pode ser utilizado para escrever procedimentos e catálogos manuais para museus; estabelecer exigências de informações para sistemas automatizados ou baseados em papel; desenvolver elementos de documentação da política de administração de coleções; prover indicadores de desempenho e padrões de serviço de qualidade para documentação de museus e justificar ofertas de recursos para apoiarem a documentação.

\subsubsection{AMICO (http://www.amico.org)}

O Art Museum Image Consortium (AMICO) é um exemplo de como os museus têm colaborado para facilitar o acesso a documentação digital de suas coleções para fins educacionais.

O AMICO é uma organização de instituições de coleções de arte sem fins lucrativos. Ele foi fundado em 1997 com 22 museus dos Estados Unidos e Canadá, através de um processo de planejamento colaborativo para expandir o uso educacional das coleções dos museus de arte de forma digital. Atualmente, já possui 39 instituições e continua crescendo.

Segundo Trant et al. (2002), os membros do AMICO têm cadastrado suas coleções para criar uma biblioteca digital conhecida como The AMICO Library, que oferece respostas a assuntos de museus e educadores em um ambiente digital.

A documentação digital incluída na biblioteca do AMICO é seguida por uma especificação de dados. Cada trabalho é representado por um registro de catálogo textual. O padrão adotado para o cadastro está baseado na Categories for the Description of Works of Art (CDWA). Cada membro mapeia os campos de seu sistema local para o dicionário de dados do AMICO. O AMICO compila a documentação e gera índices para facilitar a recuperação das informações.

O dicionário de dados do AMICO na versão 1.3 está disponível para download em http://www.amico.org/AMICOkibrary/dataDictionary.html. Ele define os campos usados no registro dos catálogos e está dividido em 14 categorias identificadas por perguntas do tipo: Como é chamado? Quem criou? Onde ele foi feito? Cada categoria possui campos relacionados que descrevem o trabalho de arte do registro. A maior parte das categorias só tem sentido para obras de arte, já que o AMICO é um consórcio de museus de arte. 


\subsubsection{CHIN (http://www.chin.gc.ca/)}

O Canadian Heritage Information Network (CHIN) e os museus do Canadá trabalham juntos para armazenar e apresentar o conteúdo digital para o público, promovendo educação e entretenimento.

O Guia CHIN para padrões de documentação de museus inclui informações de:

- padrões e diretrizes de interesse para museus;

- projetos atuais que envolvem pesquisa de padrões e implementação; e

- organizações responsáveis por pesquisa de padrões e desenvolvimento.

O padrão CHIN é baseado no Dublin Core e inclui controle de vocabulário para projetos de aprendizagem.

\subsubsection{Projeto MIDIIS (http://www.cimi.org)}

O Museum Initiative for Digital Information Interchange Standards (MIDIIS) é uma iniciativa apoiada pelo programa CIMI.

O MIDIIS utiliza metadados para promover a interoperabilidade e troca de informações entre museus. É uma iniciativa que deseja entender o universo de informações dos museus e que tipo de informações pertencente a eles pode ser compartilhada e re-utilizada por uma variedade de aplicações.

Este projeto envolve o Dublin Core e preocupa-se em saber como os museus podem efetivamente prover acesso as informações que eles criam e mantém em formato digital como, por exemplo, informações sobre coleções, exibições, pessoas, atividades educacionais, publicações e eventos.

O objetivo é estudar as diferentes estruturas de museus e testar o XML como sintaxe para representar a identificação semântica das informações e desenvolver uma DTD para representar diferentes tipos de informações. Este projeto ainda encontra-se em desenvolvimento.

\subsection{Mapeamento dos padrões}

Com base nos estudos realizados, foram definidos dois tipos de mapeamentos entre os padrões para facilitar a compreensão. O primeiro mapeamento foi feito a nível de categorias considerando os padrões AMICO e LOM, já que são os únicos que foram agrupados por meio de categorias. A tabela 1 representa este mapeamento.

Tabela 1 - Mapeamento das Categorias dos Padrões LOM e AMICO

\begin{tabular}{|l|l|}
\hline \multicolumn{1}{|c|}{ IEEE LOM } & \multicolumn{1}{c|}{ AMICO } \\
\hline Geral & $\begin{array}{l}\text { Identificador único } \\
\text { O que é isso? } \\
\text { Como é chamado? }\end{array}$ \\
\hline Ciclo de vida & $\begin{array}{l}\text { Quem criou? } \\
\text { Quando foi feito? } \\
\text { Controle de versões? }\end{array}$ \\
\hline Dados técnicos & O que ele se parece? \\
\hline Educacional & \\
\hline Direitos & Quem possui? \\
\hline Relações & Como que ele está relacionado? \\
\hline Anotações & \\
\hline
\end{tabular}




\begin{tabular}{|l|l|}
\hline Classificação & \\
\hline Meta-Metadados & Quem documentou? \\
\hline & Onde foi feito? \\
\hline & Sobre o que é? \\
\hline & O que isso significa? \\
\hline & Quem possui? \\
\hline
\end{tabular}

É possível verificar na tabela que as categorias geral e ciclo de vida do padrão LOM foram mapeadas, cada uma, para mais de uma categoria do padrão AMICO. Adicionalmente, ambos os padrões possuem categorias que não são contempladas no outro padrão, como é o caso da categoria "O que isso significa?" do padrão AMICO, a qual está fortemente ligada ao contexto de aplicação - museus de arte.

O segundo mapeamento desenvolvido é um pouco mais complexo, já que visa mapear os elementos de dados tomando como ponto de referência os 15 elementos do padrão Dublin Core. Para este mapeamento foram selecionados os padrões de acordo com o contexto de suas aplicações: DC, LOM e AMICO. O mapeamento dos padrões DC e LOM pode ser encontrado em Yin et al. (2003). Por essa razão, não será apresentado na tabela. Adicionalmente, o padrão CHIN não será apresentado no mapeamento pois sua definição foi totalmente baseada no Dublin Core. Neste sentido, todos os elementos do DC foram utilizados no CHIN, juntamente com a criação de especificações mais detalhadas.

Tabela 2 - Mapeamento dos elementos de dados dos padrões LOM e AMICO

\begin{tabular}{|l|l|}
\hline \multicolumn{1}{|c|}{ IEEE LOM } & \multicolumn{1}{c|}{ AMICO } \\
\hline General.Identifier & AMICO Identifier \\
\hline General.Title & Object-Title-Name \\
\hline General.Language & \\
\hline General.Description & Physical Description \\
\hline General.Keyword & \\
\hline General.Coverage & Object-Type \\
\hline Educational.LearningResourceType & Creation-Dates \\
\hline LifeCycle.Contribute.Date & Creator-Name-Text \\
\hline $\begin{array}{l}\text { LifeCycle.Contribute.Entity when } \\
\text { LifeCycle.Contribute.role="Author" }\end{array}$ & \\
\hline $\begin{array}{l}\text { LifeCycle.Contribute.Entity whin } \\
\text { LifeCycle.Contribute.role }\end{array}$ & \\
\hline $\begin{array}{l}\text { LifeCycle.Contribute.Entity when } \\
\text { LifeCycle.Contribute.role="Publisher" }\end{array}$ & \\
\hline Technical.Format & Classification-Term \\
\hline Rights.Descriotion & Rights/Copyright \\
\hline Relation.Resource.Description & Related Works of Art \\
\hline $\begin{array}{l}\text { Relation.Resource when } \\
\text { Relation.Kind="IsBasedOn" }\end{array}$ & \\
\hline
\end{tabular}

3 DATA/MCT - paDrão de metAdados para objeTos de Aprendizagem de Museus de Ciências e Tecnologia 
O padrão DATA/MCT foi definido para armazenar informações semânticas de objetos de aprendizagem de museus de ciências e tecnologia. Ele especifica um esquema de dados conceitual que define a estrutura do metadado para o objeto de aprendizagem. Neste padrão, assim como no LOM, um objeto de aprendizagem é definido como uma entrada digital ou não-digital, que pode ser usada para aprendizagem formal, não-formal e informal, educação e treinamento.

No contexto de museus, objetos com entrada digital são aqueles que necessitam do computador para sua utilização, como, por exemplo, um jogo educacional computadorizado. Por sua vez, os objetos com entrada não-digital são caracterizados pelos experimentos, interativos ou não, disponíveis na área de exposição do museu ou em seu acervo. Tais objetos necessitam da presença do aprendiz no local em que se encontram a fim de possibilitar o contato direto sem o uso do computador, como, por exemplo, dioramas e animais empalhados. No entanto, muitas vezes, os objetos com entrada não-digital podem possuir uma representação computadorizada que contenha algumas informações, textuais ou não, sobre o mesmo, permitindo que o aprendiz se familiarize antes da experimentação direta. Esse tipo de recurso é muito utilizado em museus virtuais que permitem ao visitante definir sua programação no museu antes da visitação.

Como ponto de partida para a definição do padrão de metadados foi necessário, primeiramente, a identificação dos diferentes tipos de informações disponíveis em museus de ciências e tecnologia. Tal estudo considerou como base objetos de aprendizagens digitais e não-digitais de diversos museus, como será apresentado na próxima seção.

\subsection{Mapeamento dos objetos digitais e não-digitais de museus}

A identificação dos tipos de objetos presentes em museus de ciências e tecnologia foi realizada com estudos em museus nacionais e internacionais, muitos com base em relatórios fotográficos elaborados a partir de visitas com fins de pesquisa. Entre os museus encontram-se: Museu Zoobotânico Augusto Ruschi (Muzar/UPF); Museu de Ciências e Tecnologia (MCT/PUCRS); Science Museum of Boston; Science Museum (Londres) e The Natural History Museum (Londres).

Pode-se observar claramente que existe uma gama muito grande de experimentos nos museus e para facilitar a identificação foi necessária a elaboração de uma classificação com base no tipo de representação do objeto e na sua complexidade. Para isso foram definidas três classes distintas:

a) objetos com representação digital: são sistemas unicamente computacionais que não envolvem nenhum manuseio mecânico além do computador e seus periféricos. Exemplos: as multimídias apresentadas em quiosques para expor informações adicionais aos demais experimentos, museus virtuais, muitos disponíveis apenas na Web e publicações em geral.

b) objetos com representação não-digital: este tipo de experimento não utiliza nenhum tipo de ambiente computacional, sendo basicamente mecânicos.

c) objetos com representação digital e não-digital: a interação entre o aprendiz e o experimento ocorre através de dispositivos ligados ao experimento e a um computador não visível ao visitante. O aprendiz irá interagir com componentes adicionais ao computador como, por exemplo, maquetes e peças históricas. As informações solicitadas pelo aprendiz, através da interação, podem ser apresentadas em vídeo e em ambientes não computacionais. Exemplos: experimentos onde o computador é responsável 
pela comunicação entre o meio mecânico e as informações apresentadas através de dispositivos computacionais.

Em qualquer uma das classes acima pode-se gerar uma instância para o objeto no padrão de metadados definido. No entanto, na classe b o objeto terá representado apenas suas informações semânticas, não sendo possível sua visualização via computador. Para o aprendiz interagir com objetos classificados nesta categoria, deverá visitar o museu. Por sua vez, na categoria c apenas parte do experimento pode ser visualizado, se houver sentido sem a parte mecânica.

No entanto, o aprendiz poderá recuperar, através de consulta, os objetos que melhor respondem suas dúvidas necessitando ou não de sua presença no local físico em que o objeto está exposto. É importante salientar que com a interoperabilidade de dados proporcionada pelo uso deste padrão, o aprendiz poderá ter como retorno, na maioria das vezes, mais de um museu, ficando a seu critério a busca especifica por uma localização.

\subsection{Descrição do DATA/MCT}

O padrão DATA/MCT foi criado a partir de elementos definidos nos padrões CHIN, LOM, AMICO e DC. Também foram criados novos elementos com base no levantamento preliminar dos objetos de aprendizagem apresentado na seção anterior.

\subsubsection{Estrutura Básica do Metadados}

Os elementos de dados são responsáveis por descrever os objetos de aprendizagem e são agrupados em categorias, conforme o padrão LOM. O DATA/MCT consiste em 8 dessas categorias:

a) A categoria Geral agrupa informações gerais que descrevem o objeto de aprendizagem em sua totalidade, como o título, tipo, local de armazenamento e palavras-chave do objeto. Para a criação desta categoria tomou-se como base os padrões LOM, AMICO e CHIN.

b) A categoria Histórico descreve toda a evolução do objeto desde a sua criação até o estado atual de desenvolvimento. Nesta categoria é possível saber se o objeto já está concluído, em que versão se encontra e quais foram as pessoas ou instituições que contribuíram para o seu desenvolvimento. O padrão LOM serviu como base para a criação desta categoria.

c) A categoria Aspectos Técnicos descreve requerimentos e características técnicas do objeto de aprendizagem. Esta categoria é que auxilia o aprendiz no download e na instalação do objeto, identificando tamanho e requerimento. Os padrões LOM, AMICO e CHIN servirão como base para a criação desta categoria.

d) A categoria Aspectos Pedagógicos descreve as características educacionais e pedagógicas do objeto de aprendizagem, apresentado, entre outros, o nível de dificuldade do objeto, o público a que se destina, o nível de interatividade e os prérequisitos. Para a criação desta categoria tomou-se como base os padrões CHIN e LOM.

e) A categoria Direitos descreve os direitos de propriedades intelectuais do objeto. Os padrões LOM, AMICO e CHIN serviram como base para a criação desta categoria.

f) A categoria Relações define o relacionamento do objeto com outros objetos, se houver, demonstrando o tipo de relacionamento existente baseado nas definições do Dublin Core. Foi baseado nos padrões LOM e AMICO. 
g) A categoria Documentação descreve os aspectos relacionados com a documentação do objeto no padrão, quem definiu, como e quando. Os padrões AMICO e LOM serviram como base para a sua criação.

h) A categoria Classificação descreve o objeto segundo um outro sistema de classificação que pode ser utilizado como um sistema alternativo e complementar para o padrão de metadados. Para essa categoria utilizou-se como padrão base o LOM.

\subsubsection{Elementos de dados}

As categorias agrupam elementos de dados. Os elementos podem ser elementos simples ou uma agregação de elementos de dados, que formam nodos folhas em uma hierarquia, como no padrão LOM e AMICO. Quando trata-se de um elemento agregado, somente os nodos folhas possuem valores definidos.

Cada elemento de dados do padrão DATA/MCT define:

a) número: utilizado para identificação do elemento, distinguindo elementos simples de agrupamentos. Exemplo: o elemento 3.6 (agrupamento) possui dois componentes: 3.6.1 (elemento simples) e 3.6.2 (elemento simples). Neste caso, somente os elementos simples possuem um valor associado.

b) padrão base: aponta o(s) padrão(ões) de origem do elemento. Pode possuir como valores: LOM; DC; CHIN; AMICO; Todos (quando todos os padrões possuem tal elemento definido), Novo (quando o elemento foi criado especificamente para o contexto desta aplicação) e Adaptado (quando aparecer a palavra adaptado antes do nome do padrão significa que o padrão base possui tal elemento, mas foram realizadas modificações para se adequar ao contexto da aplicação). É possível também uma combinação destes padrões.

c) nome: o nome pelo qual o elemento é referenciado.

d) descrição: uma descrição sobre o elemento.

e) valores possíveis: o conjunto de valores possíveis para o elemento de dados.

f) exemplos: exemplos ilustrativos do elemento, para auxiliar na catalogação.

É importante deixar claro que este padrão não objetiva substituir o banco de dados das coleções em que constam informações como, por exemplo, coleta, coletor, filo, espécie e família. O padrão de metadados busca unicamente cadastrar as informações com objetivo educacional, preocupando-se em apresentar, além de outros, o melhor modo de utilizar tal objeto. O elemento 1.3.1 denominado catálogo é responsável por fazer um link entre o objeto que está sendo descrito e o seu registro da base de dados da coleção, quando houver.

O padrão possui 54 elementos simples definidos e está atualmente sendo validado junto ao Museu Zoobotânico Augusto Ruschi (Muzar) - UPF, onde será implantado inicialmente o ambiente de consulta.

\section{Conclusões}

A construção do DATA/MCT tem relevância tanto a nível científico e tecnológico como educacional, trazendo contribuições tanto para a educação quanto para o desenvolvimento de sistemas para museus e para a pesquisa.

Do ponto de vista educacional, o ambiente visa ser uma poderosa ferramenta de consulta e estudos, auxiliando alunos e professores na busca semântica de informações para ajuda-los na construção de seu conhecimento. 
Do ponto de vista do desenvolvimento de sistemas computacionais voltados à aprendizagem em museus, o ambiente contribui para a inovação de uma linha educacional pouco explorada em ambientes educacionais para museus.

Do ponto de vista da pesquisa, o ambiente enfoca a questão da adaptação das tecnologias computacionais para apoiar a aprendizagem em museus, tornando ainda mais forte a ligação entre museus e informática.

Como o ambiente será implantado inicialmente no Muzar, com a informatização de seus objetos de aprendizagem, o museu terá condições de melhorar a qualificação da linguagem museográfica, o que possibilitará facilidade de acesso à informação. $O$ Muzar terá condições de transmitir as informações restritas ao mundo científico, para uma comunidade sedenta de informações sobre o Patrimônio Natural da região e sensibilizado para agir pela sua preservação. A citação a seguir expressa o papel dos museus: "Ao longo dos séculos, o museu caixa-forte de uma coleção reunida por um único homem, encerrada para exposição em um altar esplendoroso para deleite do público, cessa de ser um refúgio nostálgico, tornando-se um serviço público, museuescola ou museu-laboratório, que, abrindo-se, transpõe seus muros e torna-se um museu aberto onde a comunidade se encontra e se expressa." (Giraudy \& Boulhet, 1990)

O Muzar, em vários momentos, foi um museu-escola ou museu-laboratório. Envolvendo seu visitante em uma exposição interativa ou observando a natureza em uma trilha interpretativa, vivenciou a aprendizagem acontecendo.

Integrando a nova visão de museu ao movimento que a computação e a energia podem oferecer é possível disponibilizar com mais facilidade a informação. Informação esta, que muitas vezes fica omitida no íntimo da peça museológica, mas que interagindo com os atuais nobres meios de comunicação - fala e informatização - é possível transmitir conhecimento e influenciar a sensibilidade para a valorização dos Patrimônios Natural e Cultural. Neste momento, inserir a informatização dos objetos de aprendizagem do Muzar passa a ser, a principal forma de ampliar sua criação, trazendo para o museu a chance de atingir ainda mais a sociedade.

Como continuidade do trabalho serão implementados mapas de tópicos com uma classificação especifica e todo o ambiente de consulta será disponibilizado via Web.

\section{Agradecimentos}

Agradecemos ao Conselho Nacional de Desenvolvimento Científico e Tecnológico CNPq - pela aprovação desse projeto através do edital MCT/SECIS/CNPq n ${ }^{\circ}$ 07/2003.

\section{Referências Bibliográficas}

CHIN. Canadian Heritage Information Network. 2002. http://www.chin.gc.ca/

Giraudy, Danièle, Bouilhet, Henri. O museu e a vida. Trad. Jeanne France Filiatre Ferreira da Silva. Rio de Janeiro: Fundação Nacional Pró-memória; Porto Alegre: Instituto Estadual do Livro --RS; Belo Horizonte: UFMG, 1990.

IEEE Learning Technology Standards Committee (LTSC) "Draft Standard for Learning Object Metadata IEEE 1484.12.1-2002. http://ltsc.ieee.org/

Trant, J. , Bearman, D.. 2002. Educational Use of Museum Multimedia the AMICO Library. Art Libraries Journal, Volume 27, No. 2.

Yin, Z., Xu, Z., e Saddik, Ab. 2003. Study of Metadata for Advanced Multimedia Learning Objects. In: CCECE2003. IEEE. Montreal. 